\title{
Higher Incidence of Complete Lateral Meniscal Root Tears in Revision Compared With Primary Anterior Cruciate Ligament Reconstruction
}

\author{
Brian J. Vial, M.D., Andrew B. Kohner, M.D., and Theodore B. Shybut, M.D.
}

\begin{abstract}
Purpose: To evaluate the incidence of complete lateral meniscal posterior root tears (LMPRTs) repaired at revision as compared with primary anterior cruciate ligament (ACL) reconstruction (PACLR) and to determine whether other demographic or surgical characteristics were associated with LMPRTs needing repair. Methods: A chart review was performed to identify the PACLR and revision ACL reconstruction (RACLR) cohorts. Demographic and surgical characteristics were recorded. Cases with concurrent lateral meniscal posterior root repair were identified. Cases were classified as acute ( $<5$ months) or chronic ( $>5$ months) based on the time from reported injury to surgery. Tunnel malposition in revision cases was recorded if either tunnel or both tunnels were malpositioned on radiographs and magnetic resonance imaging. Results: Data from 167 cases, 140 PACLR and 27 RACLR cases, were included. The cohorts had similar demographic characteristics including age, sex, and lateral meniscal injury. The overall incidence of lateral meniscal root repair in ACL reconstruction patients was $12.6 \%$ (21 of 167 patients). The incidence of LMPRT repair was $7.1 \%$ (10 of 140 patients) in the PACLR cohort versus 40.7\% (11 of 27 patients) in the RACLR cohort. The revision cohort was significantly more likely to have a chronic injury $(66.7 \%$ [18 of 27 patients] vs $31.4 \%$ [44 of 140 patients]). The most significant predictor of concurrent lateral meniscal posterior root repair was RACLR versus PACLR for both univariate and multivariate logistic regression analyses $\left(\chi^{2}=20.603 ; P<.0001\right.$; odds ratio, 13.887; 95\% confidence interval, 1.531-125.993). Analysis of tunnel positions for the revision group revealed that PACLR tunnel malposition was a significant predictor of LMPRTs $\left(\chi^{2}=4.91, P=.027\right)$. Conclusions: Complete LMPRTs warranting repair are encountered with a significantly greater frequency at RACLR as compared with PACLR. The overall incidence of LMPRT repair at RACLR is high. In this cohort, LMPRT repair in RACLR cases was associated with tunnel malposition of the PACLR. Level of Evidence: Level III, retrospective cohort study.
\end{abstract}

$\mathbf{T}$ he menisci are critical stabilizing structures in the knee that become especially important in the setting of anterior cruciate ligament (ACL) deficiency. ${ }^{1-4}$ The lateral meniscus, particularly its posterior root, is a secondary stabilizer to the pivot shift. Musahl et al. ${ }^{5}$ showed-and Frank et al. ${ }^{3}$ confirmed-that a lateral meniscectomy further destabilized the ACL-deficient knee during a simulated

\footnotetext{
From the Joseph Barnhart Department of Orthopedic Surgery, Baylor College of Medicine, Houston, Texas, U.S.A.

The authors report the following potential conflicts of interest or sources of funding: T.B.S. is on the editorial board of Arthroscopy; is on the social media board of the Arthroscopy Association of North America; is a committee member of the American Orthopaedic Society for Sports Medicine and American Academy of Orthopaedic Surgeons; receives clinical trial research support from Zimmer and Biomet; receives research support from Arthrex and Smith $\theta$ Nephew; is a consultant for DJO Surgical; and owns stock in ROMTech, outside the submitted work. Full ICMJE author disclosure forms are available for this article online, as supplementary material.

This article was a podium presentation (under the title "Incidence of Lateral Meniscus Root Tears in Revision Versus Primary ACL
}

pivot-shift maneuver. Recently, Shybut et al. ${ }^{6}$ showed that disruption of the posterior root of the lateral meniscus further destabilized ACL-deficient knees during rotational loading.

Studies have shown that combined ACL and meniscal injuries are common and frequently involve the posterior horn of the lateral meniscus. ${ }^{7,8}$ Prior studies have reported a $7 \%$ to $14 \%$ incidence of lateral meniscal

\footnotetext{
Reconstruction") at the 37th Annual Meeting of the Arthroscopy Association of North America, Chicago, IL, April 26-28, 2018.

Received March 14, 2020; accepted September 25, 2020.

Address correspondence to Theodore B. Shybut, M.D., Joseph Barnhart Department of Orthopedic Surgery, Baylor College of Medicine, 7200 Cambridge St, Ste 10A, Houston, TX 77030,U.S.A. E-mail: shybut@bcm.edu

(C) 2021 THE AUTHORS. Published by Elsevier Inc. on behalf of the Arthroscopy Association of North America. This is an open access article under the CC BY-NC-ND license (http://creativecommons.org/licenses/by-nc-nd/4.0/). 2666-061X/20304

https://doi.org/10.1016/j.asmr.2020.09.027
} 

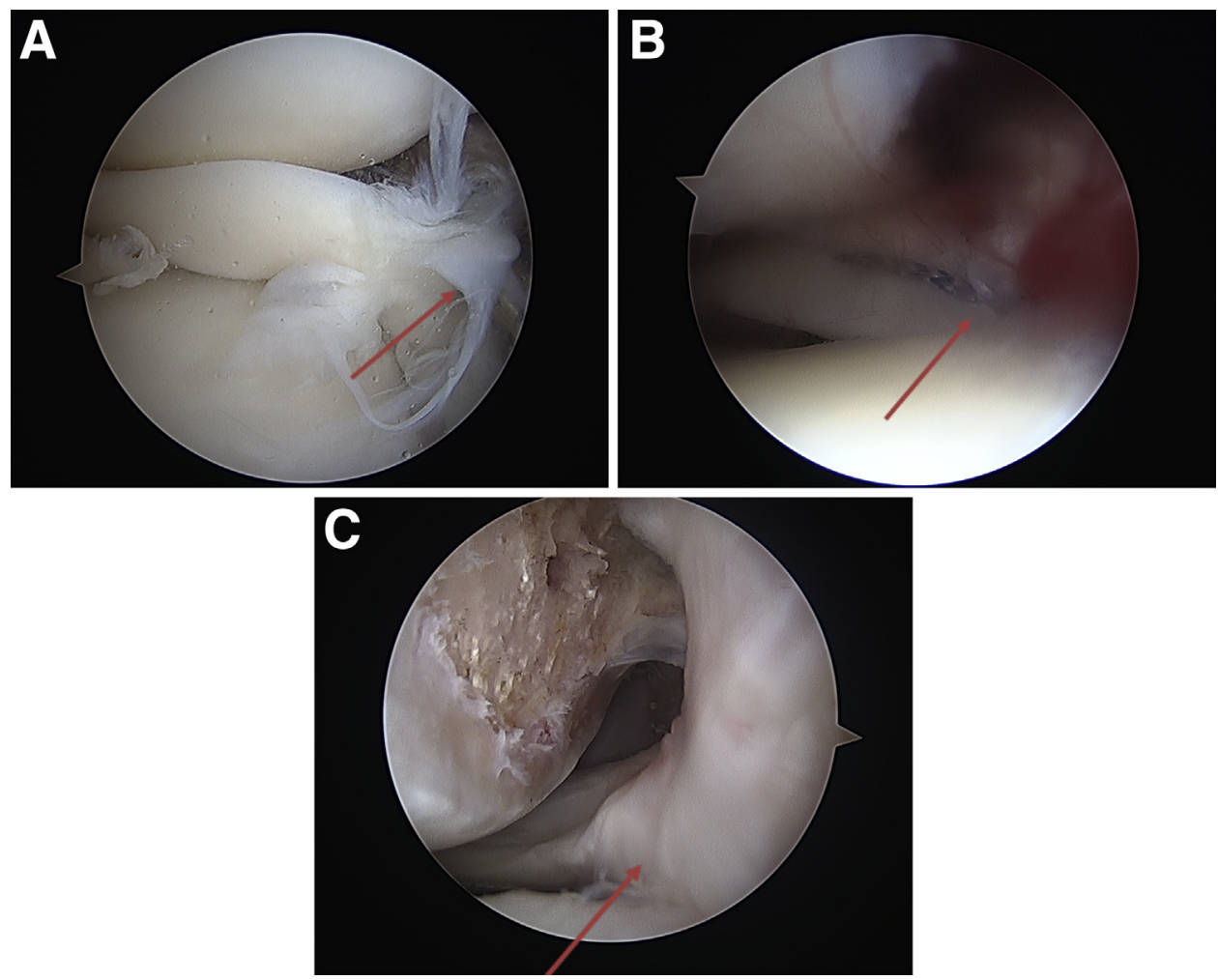

Fig 1. (A) LaPrade type 3 root tear (right knee). The arrow points at the lateral meniscal posterior root tear (LMPRT). (B) Root repair performed during first stage of 2-stage revision anterior cruciate ligament reconstruction (right knee). The arrow points at the LMPRT repair using the transtibial technique. (C) Healed root repair seen during second stage of revision anterior cruciate ligament reconstruction (right knee). The arrow points at the healed LMPRT.

posterior root tears (LMPRTs) with ACL ruptures. ${ }^{9-11}$ One study associated male sex and a contact mechanism of injury with LMPRTs in patients with ACL injury. ${ }^{12}$ These tears are frequently located within the posterior horn at or near the meniscal root. ${ }^{9,10}$

Comparisons of associated intra-articular pathology between revision ACL reconstruction (RACLR) and primary ACL reconstruction (PACLR) are few. Borchers et al. ${ }^{13}$ reported overall meniscal and chondral injury rates in the MOON (Multicenter Orthopaedic Outcomes Network) versus MARS (Multicenter ACL Revision Study) cohorts. Mitchell et al. ${ }^{14}$ similarly characterized associated lesions and radiographic findings in PACLR versus RACLR cases. The purposes of this study were to evaluate the incidence of complete LMPRTs repaired at revision as compared with PACLR and to determine whether other demographic or surgical characteristics were associated with LMPRTs needing repair. It was hypothesized that an increased incidence of lateral meniscal root tears would be seen at RACLR compared with PACLR and that other patient or surgical factors may correlate with any observed difference in incidence.

\section{Methods}

This was a retrospective analysis of patients treated by the senior author (T.B.S.). After institutional review board approval was obtained (protocol H-41053), records for patients identified by Current Procedural
Terminology code 29888 between July 2013 and March 2018 were reviewed. The inclusion criteria for the study were patients aged 18 years or older who underwent PACLR or RACLR. Patients who underwent isolated ACL repairs and single-bundle reconstructions for partial tears were excluded. Additional exclusion criteria were PACLR or RACLR in the setting of multiligamentous knee injury, staged or concurrent osteotomy in association with ACL surgery, and patient age younger than 18 years. The primary indication for ACL reconstruction (ACLR) and RACLR was treatment of functional knee instability during activity. The presence of a concurrent repairable meniscal tear was a common additional consideration.

Chronic ACL injury was defined as definitive treatment occurring greater than 5 months after injury. This definition was based on the American Academy of Orthopaedic Surgeons ACL clinical practice guideline, which recommends ACLR within 5 months of injury to decrease the risk of secondary meniscal tears. ${ }^{15}$ The presence of an LMPRT was recorded if a lateral meniscal root repair was performed in conjunction with PACLR or RACLR. The senior author's technique for meniscal root repair ${ }^{16,17}$ and his approach to RACLR ${ }^{18}$ have been previously described. Figure 1 shows a LaPrade type 3 root tear, root repair performed during the first stage of a 2-stage RACLR, and the healed repair seen during the second-stage RACLR. 
Table 1. Revision Graft Choice and Tunnel Position

Revision ACLR $(\mathrm{n}=27)$

\begin{tabular}{lc}
\hline Graft type & \\
Bone-tendon-bone autograft & $7(29.6)$ \\
Hamstring autograft & $0(0.0)$ \\
Quadriceps tendon autograft & $9(33.3)$ \\
Allograft & $3(11.1)$ \\
Unknown & $9(33.3)$ \\
Tunnel position & $18(66.7)$ \\
Acceptable & \\
Malposition & \\
\hline NOTE. Data are presented as number (percentage). & \\
ACLR, anterior cruciate ligament reconstruction. &
\end{tabular}

Demographic data (age at the time of injury and sex) and surgical information (primary or revision reconstruction, graft type, date of injury, date of surgery, magnetic resonance imaging [MRI] findings, and findings at the time of surgery, comprising medial meniscal injury, lateral meniscal injury, articular cartilage lesions, and tunnel position or malposition) were recorded. The senior author routinely meticulously inspects and probes the meniscal roots during ACL surgery. Lateral meniscal posterior root repair was performed if a complete, unstable root or root-equivalent tear was found at arthroscopy. In all such cases, transtibial repair was performed (Fig 1). ${ }^{16}$ All complete LMPRTs encountered during cases were repaired ${ }^{17}$; no complete or unstable LMPRTs were treated with debridement or partial meniscectomy. Other stable, partial, or longitudinal tears that involved or were near the lateral meniscal posterior root and were repaired with suture through capsule, rasped and left in situ, or debrided were recorded as lateral meniscal tears but were excluded from the LMPRT groups in this study because these tears did not warrant formal root repair. As is routine practice, determinations about tunnel malposition and revision tunnel position for RACLR were made at the time of planning revision surgery. For this study, imaging was retrospectively reviewed by the senior author, and tunnel malposition was recorded if present. Prior studies have shown that appropriate tunnel position can be assessed on imaging, ${ }^{19}$ but the authors concede that interobserver agreement can be variable. ${ }^{20}$ Of note, outside surgeons performed all but 1 of the PACLRs in the revision cohort.

The statistical significance of cohort characteristics was tested using multivariate logistic regression to identify risk factors for LMPRTs and calculate odds ratios (ORs). The Pearson $\chi^{2}$ test was used to perform univariate testing and to analyze the contribution of tunnel position to the incidence of LMPRTs in revision cases. The level of significance, OR, and 95\% confidence interval were calculated for each variable. Statistics were calculated using Microsoft Excel.

\section{Results}

We reviewed 186 charts for inclusion; 167 patients met the inclusion criteria. The charts were divided into 140 in the primary cohort and 27 in the revision cohort. In both cohorts, the mean age at the time of injury was 31 years, with the median skewing slightly younger in the primary cohort, at 28 years versus 31 years in the revision cohort. In both cohorts, men comprised more than $59 \%$ of patients, roughly matching the demographic characteristics of sports participants in the United States. In terms of injury characteristics, the revision cohort had a significantly higher incidence of chronic injury than the primary cohort: $66.7 \%$ (18 of 27 patients) versus $31.4 \%$ (44 of 140 patients). The overall incidence of lateral meniscal injury was higher in the revision cohort, at $66.7 \%$ (18 of 27 patients) versus $57.1 \%$ (80 of 140 patients) in the primary cohort, but was not significantly different $(P=.36)$.

Primary surgery ACL graft type and tunnel position rating for the revision group are listed in Table 1. The most common pattern of tunnel malposition encountered was combined tibial and femoral tunnel malposition, with the femoral tunnel positioned toward the roof of the notch rather than the lateral wall and the tibial tunnel relatively posterior in the ACL tibial footprint. Of note, 3 patients ( $11 \%$ of the revision cohort) required bone grafting of the prior tunnels and staged ACL revision reconstruction for widened $(\geq 14 \mathrm{~mm})$ malpositioned tibial tunnels.

The overall incidence of lateral meniscal root repair in ACLR patients was $12.6 \%$ ( 21 of 167 patients). The incidence of LMPRT repair was $7.1 \%$ (10 of 140 patients) in the PACLR cohort versus $40.7 \%$ (11 of 27 patients) in the RACLR cohort. On univariate testing, RACLR was significantly associated with LMPRTs $\left(\chi^{2}=\right.$ 20.603, $P<.0001)$. The calculated OR showed that RACLR was significantly associated with lateral meniscal root injuries (OR, 13.887; 95\% confidence interval, 1.531-125.993). Analysis of the revision group revealed an equal demographic distribution between the group without LMPRTs and the group with LMPRTs. Tunnel malposition was a significant predictor of LMPRTs in the revision group ( 10 of 11 patients [90.9\%] with LMPRTs had malpositioned tunnels on preoperative radiographs; $\left.\chi^{2}=4.91 ; P=.027\right)$. A post hoc power analysis was performed using the incidence of LMPRTs found, along with the number of patients in each cohort, which confirmed sufficient power $(98 \%)$ with an $\alpha$ value of .05.

\section{Discussion}

There are 2 important findings in this study: First, LMPRTs requiring repair were significantly more prevalent at revision as compared with PACLR. This finding confirmed the study's hypothesis. Second, 
LMPRTs in RACLR cases were associated with primary ACL tunnel malposition in the study's revision cohort. Thus, these findings appear to fit with the contemporary understanding of the biomechanical interplay between the lateral meniscal posterior root and the ACL.

The first major finding was that the rate of LMPRT repair in RACLR cases was significantly greater than in PACLR cases. The PACLR LMPRT repair incidence is consistent with findings of prior studies, which have reported $7 \%$ to $14 \%$ rates of lateral meniscal posterior root injuries concurrent with ACL tears. ${ }^{9-11}$ However, some of these were imaging-based studies and did not differentiate partial lesions or stable tears that may not need repair as compared with unstable, complete tears that were repaired in our patients. A study by Ahn et $\mathrm{al}^{21}$ reported a $6.95 \%$ incidence of concomitant LMPRTs that required repair at the time of ACLR.

The second major finding was that LMPRTs in revision ACL surgery were associated with primary ACL tunnel malposition in this study's revision cohort. A prior study has shown that tunnel malposition leads to an increased rate of cartilaginous and meniscal pathology. ${ }^{22}$ It is possible that altered pivot-shift biomechanics in ACL-reconstructed knees with malpositioned tunnels may be a factor in these root tears. Even though ACLR techniques have evolved, technical failures, albeit less obvious, still may occur.

These clinical data corroborate the concept that the lateral root is a secondary stabilizer of the pivot shift, particularly if one assumes that the revision ACL knees in this study have a greater cumulative exposure to pathologic pivot-shift loading. DePhillipo et al. ${ }^{23}$ recently reported the results of meniscal repairs performed during staged RACLRs. Although their study specifically looked at staged RACLRs, they also reported an increased incidence of posterior lateral meniscal root tears in the revision versus primary cohort $(20.2 \%$ vs $12.2 \%)$, which is corroborated by the findings reported in our study.

Recognizing LMPRT injuries prior to surgery is important for both the patient and the surgeon. For the patient, a meniscal root repair may significantly alter his or her rehabilitation protocol because it generally entails protected weight bearing during the early postoperative weeks. In addition, medial posterior root tears have been shown to be the equivalent of a total meniscectomy, whereas repair can restore normal joint biomechanics. ${ }^{24}$ Nonoperative management of medial meniscal posterior root tears is associated with poor long-term clinical outcomes; it is possible that similar adverse effects occur when LMPRTs are not treated. ${ }^{25} \mathrm{~A}$ recent biomechanical study showed the importance of lateral meniscal posterior root repair in restoring native biomechanics in the ACL-reconstructed knee. ${ }^{26}$ For the surgeon, anticipating and/or recognizing this concurrent pathology promotes better preoperative patient counseling and ensures that the necessary resources are available for root repair at surgery. The sensitivity of MRI for detecting LMPRTs is limited. ${ }^{27}$ Krych et al. ${ }^{28}$ reported that $67 \%$ of LMPRTs were missed on preoperative MRI. Previously, surgeons have reported that many lateral meniscal tears in the region of the posterior root are small, stable lesions, which are likely to heal on their own after ACLR. ${ }^{29}$ It is important to realize that classic studies supporting this approach were undertaken in an era in which meniscal root lesions had not been characterized and understood as such. In a 10-year follow-up study on LMPRTs treated nonoperatively, Shelbourne et al. ${ }^{30}$ found mild lateral joint space narrowing compared with the control group, although there were no significant differences in functional scores. On the basis of the results of our study, the current clinical and biomechanical literature, and the contemporary understanding of lateral meniscal posterior root and ACL kinematics, repair of complete and/or unstable LMPRTs encountered during ACL surgery is advocated.

\section{Limitations}

There were limitations to this study. The study was retrospective. The patients comprised a single-surgeon cohort, so determining the presence of LMPRTs that necessitated repair was based on 1 surgeon's practice. However, this also ensured consistency of data collected and surgical techniques. There is theoretically a potential for bias in discovering LMPRTs in revision cases owing to heightened vigilance, but the senior author believes this is not the case because the suspicion for LMPRTs in primary ACL cases is paramount; the lateral root is routinely inspected for lesions because it is understood by the senior author to be at risk in all ACLinjured knees. In addition, a surgeon with expertise in RACLR may be more apt to discover and repair root tears, but this notion also highlights the importance of increasing awareness of this injury pattern.

Moreover, detailed data collection from preoperative radiology studies was not performed. Furthermore, outside surgeons performed all but 1 of the PACLRs in the revision cohort. Therefore, it cannot be said with certainty that the LMPRTs that were repaired in the revision cohort were not missed during the index procedures. There was heterogeneity among the patients regarding the timing of RACLR, although the data suggest that chronicity did not appear to be a factor for the incidence of LMPRTs. There was not sufficient power to sub-analyze the relative contributions of tibial versus femoral versus combined tunnel malposition. As noted previously, interobserver agreement on tunnel position based on imaging varies significantly. ${ }^{20}$

Patient outcomes and follow-up were not formally analyzed because the primary aim was to characterize differences in revision versus primary ACL surgery. We 
recognize that presenting outcome data would potentially enhance the impact of this study. Finally, the study reports the incidence of lateral meniscal posterior root repair. Repair in this series represented a 1:1 proxy for complete root tears because they were all repaired, but this may be a limitation when comparing our study with other literature with more inclusive criteria that include other patterns, such as longitudinal tears, incomplete radial tears, stable root tears, or small flap tears in the region of the root, or studies reporting tear incidence identified on imaging alone.

\section{Conclusions}

Complete LMPRTs warranting repair are encountered with a significantly greater frequency at RACLR as compared with PACLR. The overall incidence of LMPRT repair at RACLR is high. In this cohort, LMPRT repair in RACLR cases was associated with tunnel malposition of the PACLR.

\section{Acknowledgment}

The authors acknowledge Ryan Conyer, M.D., who helped with completing and revising the final statistical analyses.

\section{References}

1. Melrose J. The importance of the knee joint meniscal fibrocartilages as stabilizing weight bearing structures providing global protection to human knee-joint tissues. Cells 2019;8:324.

2. McNulty AL, Guilak F. Mechanobiology of the meniscus. J Biomech 2015;48:1469-1478.

3. Frank JM, Moatshe G, Brady AW, et al. Lateral meniscus posterior root and meniscofemoral ligaments as stabilizing structures in the ACL-deficient knee: A biomechanical study. Orthop J Sports Med 2017;5:2325967117695756.

4. Pike AN, Patzkowski JC, Bottoni CR. Meniscal and chondral pathology associated with anterior cruciate ligament injuries. J Am Acad Orthop Surg 2019;27:75-84.

5. Musahl V, Bedi A, Citak M, O'Loughlin P, Choi D, Pearle AD. Effect of single-bundle and double-bundle anterior cruciate ligament reconstructions on pivot-shift kinematics in anterior cruciate ligament- and meniscusdeficient knees. Am J Sports Med 201 1;39:289-295.

6. Shybut TB, Vega CE, Haddad J, et al. Effect of lateral meniscal root tear on the stability of the anterior cruciate ligament-deficient knee. Am J Sports Med 2015;43: 905-911.

7. Anderson MJ, Browning WM, Urband CE, Kluczynski MA, Bisson LJ. A systematic summary of systematic reviews on the topic of the anterior cruciate ligament. Orthop J Sports Med 2016;4:2325967116634074.

8. Tsujii A, Yonetani Y, Kinugasa K, et al. Outcomes more than 2 years after meniscal repair for radial/flap tears of the posterior lateral meniscus combined with anterior cruciate ligament reconstruction. Am J Sports Med 2019;47: 2888-2894.
9. Feucht MJ, Salzmann GM, Bode G, et al. Posterior root tears of the lateral meniscus. Knee Surg Sports Traumatol Arthrosc 2015;23:119-125.

10. Forkel P, Reuter S, Sprenker F, et al. Different patterns of lateral meniscus root tears in ACL injuries: Application of a differentiated classification system. Knee Surg Sports Traumatol Arthrosc 2015;23:112-118.

11. Krych AJ, LaPrade MD, Cook CS, et al. Lateral meniscal oblique radial tears are common with ACL injury: A classification system based on arthroscopic tear patterns in 600 consecutive patients. Orthop J Sports Med 2020;8: 2325967120921737.

12. Feucht MJ, Bigdon S, Mehl J, et al. Risk factors for posterior lateral meniscus root tears in anterior cruciate ligament injuries. Knee Surg Sports Traumatol Arthrosc 2015;23:140-145.

13. Borchers JR, Kaeding CC, Pedroza AD, et al. Intra-articular findings in primary and revision anterior cruciate ligament reconstruction surgery: A comparison of the MOON and MARS study groups. Am J Sports Med 201 1;39:1889-1893.

14. Mitchell JJ, Cinque ME, Dornan GJ, et al. Primary versus revision anterior cruciate ligament reconstruction: Patient demographics, radiographic findings, and associated lesions. Arthroscopy 2018;34:695-703.

15. American Academy of Orthopaedic Surgeons. Management of anterior cruciate ligament injuries. Evidence-based clinical practice guideline, https://urldefense.proofpoint. $\mathrm{com} / \mathrm{v} 2 / \mathrm{url}$ ? u = https-3A__www.aaos.org_globalassets_ quality-2Dand-2Dpractice-2Dresources_anterior-2Dcruciate2Dligament-2Dinjuries_anterior-2Dcruciate-2Dligament2Dinjuries-2Dclinical-2Dpractice-2Dguideline-2D4-2D24-2D 19.pdf\&d $=$ DwIGaQ\&c $=$ ZQs-KZ8oxEw0p8 1 sqgiaRA $8 \mathrm{r}=\mathrm{O}$ AgOFNgcYQ_Ug222_EnLeg8m=-2kP_ExOiH4ED6CwqV9 Ql6VF_TKGq-78ltvTtPk8uE\&s=TdQJ9uxqz5hjyNWwhIRB XEYZAV4VEMSnZAfiTqS1zWw\&e $=$. Published September 5, 2014. Accessed August 24, 2020.

16. Palisch AR, Winters RR, Willis MH, Bray CD, Shybut TB. Posterior root meniscal tears: Preoperative, intraoperative, and postoperative imaging for transtibial pullout repair. Radiographics 2016;36:1792-1806.

17. Shybut TB. How to succeed in revision anterior cruciate ligament (ACL) reconstruction. Case 4, https://urldefense. proofpoint.com/v2/url?u=https-3A__wwW.vumedi.com_ video_how-2Dto-2Dsucceed-2Din-2Drevision-2Danterior2Dcruciate-2Dligament-2Dacl-2Dreconstruction-2Dcase-2D 4_$\delta \mathrm{d}=$ DwIGaQ\&c $=$ ZQs-KZ8oxEw0p81 sqgiaRA\&r $=\mathrm{OAgOF}$ NgcYQ_Ug222_EnLeg8m=-2kP_ExOiH4ED6CwqV9Ql6V F_TKGq-78ltvTtPk8uESs=VAjER3EJAhS9tJtoecbphEIP dlBpmr3GJe33k6KAqeMSe=. Accessed August 24, 2020.

18. Shybut TB. How to succeed in revision anterior cruciate ligament (ACL) reconstruction, https://urldefense.proof point.com/v2/url?u=https-3A__www.vumedi.com_video_ how-2Dto-2Dsucceed-2Din-2Drevision-2Danterior-2D cruciate-2Dligament-2Dacl-2Dreconstruction_Ed=DwI $\mathrm{GaQ} \delta \mathrm{c}=\mathrm{ZQs}-\mathrm{KZ} 8 \mathrm{oxEw0p8} \mathrm{l}$ sqgiaRA\&r=oAgOFNgcYQ Ug222_EnLeg\&m=-2kP_ExOiH4ED6CwqV9Ql6VF_TK Gq-78ltvTtPk8uE\&s=mi3zravfYwWFychncrvAxJjmjt HmdojAyZTq-lntLfgEe $=$. Accessed August 24, 2020.

19. Dhawan A, Gallo RA, Lynch SA. Anatomic tunnel placement in anterior cruciate ligament reconstruction. J Am Acad Orthop Surg 2016;24:443-454. 
20. Matava MJ, Arciero RA, Baumgarten KM, et al. Multirater agreement of the causes of anterior cruciate ligament reconstruction failure: A radiographic and video analysis of the MARS cohort. Am J Sports Med 2015;43:310-319.

21. Ahn JH, Lee YS, Yoo JC, Chang MJ, Park SJ, Pae YR. Results of arthroscopic all-inside repair for lateral meniscus root tear in patients undergoing concomitant anterior cruciate ligament reconstruction. Arthroscopy 2010;26:67-75.

22. Chiba D, Tsuda E, Tsukada H, Iio K, Ishibashi Y. Tunnel malpositions in anterior cruciate ligament risk cartilaginous changes and bucket-handle meniscal tear: Arthroscopic survey in both primary and revision surgery. J Orthop Sci 2017;22:892-897.

23. DePhillipo NN, Dekker TJ, Aman ZS, Bernholt D, Grantham WJ, LaPrade RF. Incidence and healing rates of meniscal tears in patients undergoing repair during the first stage of 2-stage revision anterior cruciate ligament reconstruction. Am J Sports Med 2019;47:3389-3395.

24. Pache S, Aman ZS, Kennedy M, et al. Meniscal root tears: Current concepts review. Arch Bone Jt Surg 2018;6: 250-259.

25. Krych AJ, Reardon PJ, Johnson NR, et al. Non-operative management of medial meniscus posterior horn root tears is associated with worsening arthritis and poor clinical outcome at 5-year follow-up. Knee Surg Sports Traumatol Arthrosc 2017;25:383-389.

26. Tang X, Marshall B, Wang JH, et al. Lateral meniscal posterior root repair with anterior cruciate ligament reconstruction better restores knee stability. Am J Sports Med 2019;47:59-65.

27. LaPrade RF, Ho CP, James E, Crespo B, LaPrade CM, Matheny LM. Diagnostic accuracy of $3.0 \mathrm{~T}$ magnetic resonance imaging for the detection of meniscus posterior root pathology. Knee Surg Sports Traumatol Arthrosc 2015;23:152-157.

28. Krych AJ, Wu IT, Desai VS, et al. High rate of missed lateral meniscus posterior root tears on preoperative magnetic resonance imaging. Orthop J Sports Med 2018;6: 2325967118765722.

29. Shelbourne KD, Heinrich J. The long-term evaluation of lateral meniscus tears left in situ at the time of anterior cruciate ligament reconstruction. Arthroscopy 2004;20: 346-351.

30. Shelbourne KD, Roberson TA, Gray T. Long-term evaluation of posterior lateral meniscus root tears left in situ at the time of anterior cruciate ligament reconstruction. Am J Sports Med 2011 ; 39:1439-1443. 